\title{
Intravenous acetaminophen versus morphine sulfate in pain management of acute renal colic: a randomized clinical trial
}

\author{
Seyyed Hosein Montazer', Behzad Feizzadeh², Farzad Bozorgi', Seyed Mohammad Hosseininejad', Ayyoub \\ Barzegarnezhad ${ }^{2}$, Iraj Golikatir', Fatemeh Jahanian', Hasan Motamed', Hamed Aminiahidashti ${ }^{*}$
}

'Department of Emergency Medicine, Mazandaran University of Medical Science, Sari, Iran

2Department of Urology, Mazandaran University of Medical Science, Sari, Iran

\author{
Received: 16 August 2017 \\ Accepted: 18 September 2017 \\ Published online: 28 September 2017 \\ *Corresponding author: Hamed \\ Aminiahidashti, Imam Khomeini \\ Hospital, Amirmazandarani Boulevard, \\ Sari, Iran. \\ Tel: +989113540546 \\ Email: H.aminiahidashti@mazums.ac.ir \\ Competing interests: None. \\ Funding information: $T$ \\ Citation: Montazer SH, Feizzadeh \\ B, Bozorgi F, Hosseininejad SM \\ Barzegarnezhad A, Golikatir I, et al. \\ Intravenous acetaminophen versus \\ morphine sulfate in pain management \\ of acute renal colic: a randomized \\ clinical trial. Journal of Emergency \\ Practice and Trauma 2018; 4(1): 39-43. \\ doi: $10.15171 /$ jept.2017.33
}

\begin{abstract}
Objective: The main purpose in the treatment of renal colic patients in emergency department is pain management. This study aimed to compare the analgesic effects of intravenous (IV) acetaminophen and morphine sulfate in this regard.

Methods: This double blind clinical trial was conducted on $>18$ years old renal colic patients in need of pain management in emergency department. Pain severity was recorded as 15, 30, and 60 minutes before injection, and 120 minutes after injection. In addition, side effects were compared between IV acetaminophen and morphine sulfate groups using SPSS version 16.

Results: A total of 355 patients were randomly allocated to one of the treatment groups. There were no significant differences between the two groups regarding baseline characteristic of participants. There was no significant difference in the pain intensity of the groups; $15(P=0.13)$ and $30(P=0.15)$ minutes after treatment. Although, the difference in pain severity was statistically significant between the two groups; $60(P=0.02)$ and 120 $(P=0.003)$ minutes after the infusion. This was not clinically important. The prevalence of side effects in morphine group was higher than the acetaminophen group (RR: 2.14, 95\% Cl: $1.53-2.98, P<0.0001)$.

Conclusion: Based on the findings, IV morphine sulfate and acetaminophen had equal effectiveness regarding acute renal colic pain management, but considering the significantly higher frequency of side effects, IV acetaminophen seems to be a more reasonable choice in this regard.

Keywords: Renal colic, Morphine, Acetaminophen, Pain management, Emergency service
\end{abstract}

\section{Introduction}

Kidney stones are of the most prevalent urological problems suffered by about $2 \%-3 \%$ of the people (1). Evidence shows that renal colic is one of the most painful diseases that necessitates quick control of pain (2). The most prevalent symptom in acute renal colic is a sudden and severe throbbing pain that extends from the flank to the lower limb and is accompanied with nausea and vomiting in $85 \%$ of cases with microscopic hematuria, and one of its manifestations is costovertebral angle tenderness (3). The patients with such pain make up about $1 \%$ of emergency department visits (4). The main purpose in treatment of renal colic is reduction of the frequent analgesic doses as well as pain relief (5). Two groups of drugs are commonly used to cure renal colic pains, which include injectable nonsteroidal anti-inflammatory drugs (NSAIDs) and narcotic drugs, each of which has various contraindications and side effects $(6,7)$. Morphine as a strong narcotic substance is very effective in the treatment of pain caused by renal colic (8). It is highly addictive and high doses are needed to achieve analgesia (9). Opioid drugs have side effects like respiratory depression, excessive sedation, ileus and nausea and vomiting. Therefore, reduction of drug dosage leads to reduction of the side effects (10). Intravenous (IV) acetaminophen is a safe analgesic drug, which passes the blood brain barrier and reaches a high concentration in the central nervous system (CNS) and creates its analgesic effect through inhibition of cyclooxygenase and serotonin system (11). Considering the increase in availability and usage of IV acetaminophen in emergency departments, this study aimed to compare the analgesic effect of IV acetaminophen and morphine sulfate in patients with 
acute renal colic referring to the emergency department.

\section{Methods}

This double blind clinical trial was conducted on renal colic patients in need of pain management, visiting the emergency department of Imam Khomeini hospital, Sari, as the largest referral center of urology patients in northern Iran, during 2015.

The participation criteria included age over 18 years; diagnosis of renal colic based on clinical presentation and confirmation of stone or hydronephrosis on ultrasonography or spiral computed tomography (CT) scan; consent to participate in the study; and not having used pain killers or other analgesic agents before entering the emergency department. Known allergy to morphine and acetaminophen; unstable hemodynamics; history of urinary tract infection; symptoms of peritoneal irritation; drug addiction; kidney transplant; history of any anatomical abnormalities of the urinary tract; and pregnancy were among the exclusion criteria.

All patients eligible to enter the study were randomly assigned to one of the treatment groups using simple randomization method. The first group was treated with $0.1 \mathrm{mg} / \mathrm{kg}$ IV morphine, manufactured by Darou Paksh Company, Iran, in $100 \mathrm{~mL}$ normal saline; and the second group was treated with $1 \mathrm{~g}$ of IV acetaminophen manufactured by Exir Company, Iran, in $100 \mathrm{~mL}$ normal saline. The intravenous infusion lasted for 15 minutes in both groups. The packages containing the two treatment plans were coded and they were chosen for each patient randomly. Drugs were injected to the patients by emergency nurses, and the physician in charge was not aware of the type of drugs until the end of the evaluation. Pain intensity was assessed and recorded as 15, 30, and 60 minutes before injection, and 120 minutes after injection using visual analog scale (VAS) device. After 120 minutes with no appropriate response, another $1 \mathrm{mg} / \mathrm{kg}$ of morphine was injected as rescue analgesia. Successful treatment was when the patients had no pain after 120 minutes and the treatment response for patients was more than 3 scores of pain reduction on VAS. Other patients and also those who were given rescue analgesia were considered as no response to treatment and treatment failure.

The patients' demographic and clinical characteristics (age, sex, weight, hematuria, kidney stone size and the need for rescue analgesia) as well as possible side effects (dizziness, nausea and vomiting, hypotension, abdominal pain) and pain severity $(15,30$, and 60 minutes before treatment, and 120 minutes after treatment) were recorded in the data collection form by the senior emergency medicine resident in charge of patients.

All analyzes were performed by SPSS version 16. Mean \pm standard deviation (SD) or frequency and percentage were used to report the variables. Homogeneity of the explanatory variables in the two treatment groups was evaluated by chi-square test and student's $t$ test. Pain improvement percentage was analyzed by z-test for proportions. Pain scores in the two treatment groups were compared by Mann-Whitney U test and Friedman test. In this study, $P$ value $<0.05$ was considered significant.

\section{Results}

Finally, 355 patients were randomly allocated to one of the treatment groups. A total of 192 patients were treated with IV morphine sulfate and 152 with IV acetaminophen (Figure 1). There was no significant difference between the two groups regarding baseline characteristics of the participants (Table 1).

Table 2 compares the patients' pain severity between the two groups $(15,30$, and 60 minutes before treatment, and 120 minutes after treatment administration). There was no significant difference in the pain intensity of the groups, 15 and 30 minutes after the infusion. Although the difference in pain severity between the two groups, 60 $(P=0.02)$ and $120(P=0.003)$ minutes after the infusion, was statistically significant, it was not clinically important. One hundred twenty minutes after the injection, 40 patients (24\%) from morphine group needed rescue analgesia while in acetaminophen group, 50 patients (32.9\%) needed rescue analgesia $(P=0.06)$.

The most prevalent side effects in morphine and acetaminophen groups were dizziness and vomiting respectively. The drug side effects are depicted between the groups in Table 3.

The risk of dizziness in the morphine patients was significantly different from the acetaminophen patients and it was 3.8 (relative risk $[R R]=3.20,95 \% C I=1.99$ $7.25, P<0.0001)$. Ninety-two $(47.9 \%)$ of the morphine treated patients and $34(22.4 \%)$ of the acetaminophen treated patients suffered from side effects and side effect prevalence in morphine group was more than acetaminophen group (RR: 2.14, 95\% CI: 1.53-2.98, $P<0.0001)$.

\section{Discussion}

Based on the findings, IV morphine sulfate and acetaminophen had equal effectiveness regarding acute renal colic pain management, but considering the significantly higher frequency of side effects, IV acetaminophen seems to be a more reasonable choice in this regard.

In both groups, appropriate treatment response was seen after 30 minutes (the pain was reduced from severe to moderate), so the treatment response evaluation continued until 120 minutes after the infusion. In both groups of acetaminophen and morphine, pain intensity reduced to mild pain (120 minutes after treatment). Despite the extensive use of acetaminophen in different clinical conditions, its analgesic mechanism is not 


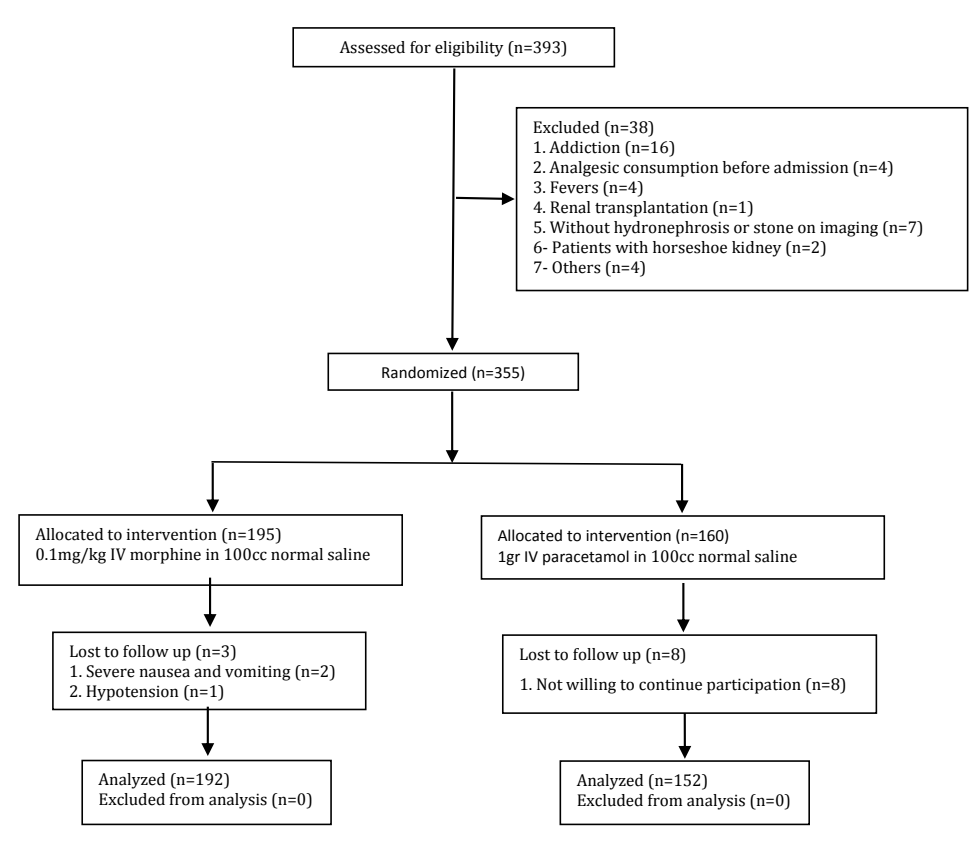

Figure 1. The study flowchart.

Table 1. Comparison of baseline characteristics of the two groups

\begin{tabular}{lccc}
\hline Variable & $\begin{array}{c}\text { Morphine } \\
(\mathbf{n}=\mathbf{1 9 2})\end{array}$ & $\begin{array}{c}\text { Acetaminophen } \\
(\mathbf{n}=\mathbf{1 5 2})\end{array}$ & $\boldsymbol{P}$ \\
\hline Sex & & & 0.31 \\
$\quad$ Male & $130(67.71)$ & $105(69.08)$ & \\
$\quad$ Female & $62(32.29)$ & $47(30.92)$ & \\
Age (y) & $41.54 \pm 13.93$ & $41.29 \pm 12.65$ & 0.86 \\
Weight(kg) & $70.43 \pm 14.27$ & $65.56 \pm 12.45$ & 0.20 \\
Hematuria & $156(81.2)$ & $121(77.60)$ & 0.33 \\
\hline Pain severity (VAS) & $8.29 \pm 1.41$ & $8.21 \pm 1.26$ & 0.56 \\
\hline
\end{tabular}

Abbreviation:VAS, visual analogue scale.

Data were presented as mean \pm standard deviation or number (\%).

Table 2. Comparison of pain severity between the two groups in 15 , 30,60 , and 120 minutes after treatment administration

\begin{tabular}{lccc}
\hline Time $(\mathbf{m i n})$ & $\begin{array}{c}\text { Morphine } \\
(\mathbf{n}=\mathbf{1 9 2})\end{array}$ & $\begin{array}{c}\text { Acetaminophen } \\
(\mathbf{n}=\mathbf{1 5 2})\end{array}$ & $\boldsymbol{P}$ \\
\hline 15 & $7.24 \pm 1.64$ & $6.98 \pm 1.46$ & 0.13 \\
30 & $5.66 \pm 1.81$ & $5.38 \pm 1.82$ & 0.15 \\
60 & $3.50 \pm 2.29$ & $4.06 \pm 2.14$ & 0.02 \\
120 & $2.38 \pm 2.06$ & $2.87 \pm 2.41$ & 0.003 \\
\hline
\end{tabular}

Data were presented as mean \pm standard deviation or number (\%).

Pain severity was reported on visual analogue scale.

Table 3. Comparison of treatment side effects between the two groups

\begin{tabular}{lccc}
\hline Time (min) & $\begin{array}{c}\text { Morphine } \\
(\mathbf{n}=\mathbf{1 9 2})\end{array}$ & $\begin{array}{c}\text { Acetaminophen } \\
(\mathbf{n}=\mathbf{1 5 2})\end{array}$ & $\boldsymbol{P}$ \\
\hline Dizziness, No. (\%) & $48(25)$ & $10(6.6)$ & 0.0001 \\
\hline Nausea and vomiting No. (\%) & $32(16.7)$ & $18(11.8)$ & 0.20 \\
\hline Hypotension, No. (\%) & $42(21.9)$ & $10(6.6)$ & 0.0001 \\
\hline Abdominal pain, No. (\%) & $2(1.0)$ & $2(1.3)$ & 0.40 \\
\hline
\end{tabular}

completely known. Acetaminophen has central inhibitory effects on cyclooxygenase and also interacts with serotonergic system (12). IV acetaminophen was first introduced in 80 countries and was approved in 2010 by the Food and Drug Administration (FDA) (13). For many years, narcotic drugs were used as the main treatment for acute renal colic, which improved it by exerting an effect on the central narcotic receptors (14). NSAIDs and opiates have several side effects like nausea and vomiting, drowsiness, respiratory depression and unexpected effects on gastrointestinal, but acetaminophen has fewer side effects in pain control after surgeries, so acetaminophen is preferred in order to prevent the side effects of opiate drugs and NSAIDs (15). In the present study, acetaminophen was used with the scant amount of $1 \mathrm{~g}$ while the higher dosage of $2 \mathrm{~g}$ of acetaminophen had a more analgesic effect (16). In some studies, there was no significant difference regarding pain reduction for 30 minutes between morphine and acetaminophen groups, and the degree of analgesia after 30 minutes was the same in both groups $(8,17)$, but the study did not continue after 30 minutes. In the present study, though after 30 minutes the pain reduction in both groups was not significantly different, the treatment continued and after 120 minutes, there was a significant difference between the two groups in terms of pain reduction. However, this statistically significant difference does not indicate clinical superiority of one drug over the other. In some studies it was shown that IV acetaminophen causes more pain reduction on VAS pain score than IV morphine and this effect continues for 60 minutes after the infusion. This difference in findings and the results from the present study may be due to the use of 
fentanyl $1 \mathrm{mg} / \mathrm{kg}$ administered intravenously 30 minutes after the beginning of the treatment process in both groups (18). Acetaminophen's analgesic effect in pain reduction of renal colic is superior to muscular piroxicam (19). Side effects such as dizziness and hypotension are transient and cause no serious problems to patients. Anyway, acetaminophen has an advantage in pain management of the patients with kidney stones, has satisfactory results in clinical use for renal colic, and is an effective drug whose treatment dosage results in fewer side effects than NSAIDs and opioids (20). Many studies confirmed the various side effects of morphine in renal colic $(21,22)$. In the present study, the side effects of morphine were twice that of intravenous acetaminophen. It seems that intravenous acetaminophen can be used as a safe and effective drug to treat the pain caused by acute renal colic.

\section{Conclusion}

Based on the findings, IV morphine sulfate and acetaminophen had equal effectiveness regarding acute renal colic pain management, but considering the significantly higher frequency of side effects, IV acetaminophen seems to be a more reasonable choice in this regard.

\section{Acknowledgments}

This paper is issued from the postgraduate thesis of Dr. Hassan Motamed and with special thanks to Mazandaran University of Medical Sciences for its support.

\section{Ethical issues}

The study design was approved by the Ethical Committee of Mazandaran University of Medical Science. The process of study and treatment was explained to all patients and verbal informed consent was taken. The patients were not required to pay any charges to participate in the study. All researchers adhered to the Helsinki Declaration during the study period. This study was registered on the Iranian registry of clinical trials website (Identifier: IRCT2017080835565N1).

\section{Authors' contributions}

SHM contributed to study concept and design. FB, FJ and HM contributed to acquisition of data. SHM, SMH and IG contributed to analysis and interpretation of data. SHM and HA contributed to drafting of the manuscript. BS and $\mathrm{AB}$ contributed to critical revision of the manuscript for important intellectual content. HM contributed to statistical analysis. FB contributed to administrative, technical, and material support. SHM and HA contributed to study supervision.

\section{References}

1. Gupta R, Gupta A, Singh G, Suri A, Mohan SK, Gupta CL. PCNL--A comparative study in nonoperated and in previously operated (open nephrolithotomy/ pyelolithotomy) patients--a single-surgeon experience. Int Braz J Urol 2011; 37(6): 739-44.

2. Smith AD, Preminger G, Badlani G, Kavoussi LR. Smith's Textbook of Endourology. 3rd ed. John Wiley \& Sons; 2012.

3. Dave C. Nephrolithiasis. Updated Dec 3, 2016. http:// www.emedicine.com/med/topic3437.htm.

4. Rezakhaniha B, Safari Nezhad MR, Markazi Moghaddam $\mathrm{N}$, Valimanesh HA, Abd Elahian M. The comparison of the efficay of commom pain management in acute renal colic. Annals of Military and Health Sciences Research 2004; 2(3): 381-5.

5. Masarani M, Dinneen M. Ureteric colic: new trends in diagnosis and treatment. Postgrad Med J 2007; 83(981): 469-72. doi: 10.1136/pgmj.2006.055913.

6. Kallidonis P, Liourdi D, Liatsikos E. Medical treatment for renal colic and stone expulsion. Eur Urol Suppl 2011; 10(5): 415-22. doi: 10.1016/j.eursup.2011.07.003.

7. Safdar B, Degutis LC, Landry K, Vedere SR, Moscovitz HC, D’Onofrio G. Intravenous morphine plus ketorolac is superior to either drug alone for treatment of acute renal colic. Ann Emerg Med 2006; 48(2): 173-81.

8. Bektas F, Eken C, Karadeniz O, Goksu E, Cubuk M, Cete Y. Intravenous paracetamol or morphine for the treatment of renal colic: a randomized, placebocontrolled trial. Ann Emerg Med 2009; 54(4): 568-74. doi: 10.1016/j.annemergmed.2009.06.501.

9. Romero V, Akpinar H, Assimos DG. Kidney stones: a global picture of prevalence, incidence, and associated risk factors. Rev Urol 2010; 12(2-3): e86-96.

10. Cattabriga I, Pacini D, Lamazza G, Talarico F, Di Bartolomeo R, Grillone G, et al. Intravenous paracetamol as adjunctive treatment for postoperative pain after cardiac surgery: a double blind randomized controlled trial. Eur J Cardiothorac Surg 2007; 32(3): 527-31. doi: 10.1016/j.ejcts.2007.05.017.

11. Remy C, Marret E, Bonnet F. State of the art of paracetamol in acute pain therapy. Curr Opin Anaesthesiol 2006; 19(5): 562-5. doi: 10.1097/01.aco.0000245285.30282.70.

12. Kesimci E, Gumus T, Izdes S, Sen P, Kanbak O. Comparison of efficacy of dexketoprofen versus paracetamol on postoperative pain and morphine consumption in laminectomy patients. Agri 2011; 23(4): 153-9. doi: 10.5505/agri.2011.86548.

13. Jahr JS, Lee VK. Intravenous acetaminophen. Anesthesiol Clin 2010; 28(4): 619-45. doi: 10.1016/j. anclin.2010.08.006.

14. Goodman Gilman A, Rall TW, Nies AS, Taylor P. Opioid analgesics and antagonists. In: Goodman and Gilman's: The Pharmacological Basis of Therapeutics. New York: McGraw-Hill; 1990. p. 485-521.

15. Elvir-Lazo OL, White PF. The role of multimodal analgesia in pain management after ambulatory surgery. Curr Opin Anaesthesiol 2010; 23(6): 697-703. doi: 10.1097/ACO.0b013e32833fad0a.

16. Juhl GI, Norholt SE, Tonnesen E, Hiesse-Provost O, Jensen TS. Analgesic efficacy and safety of intravenous paracetamol (acetaminophen) administered as a $2 \mathrm{~g}$ starting dose following third molar surgery. Eur J Pain 2006; 10(4): 371-7. doi: 10.1016/j.ejpain.2005.06.004. 
17. Morteza-Bagi HR, Amjadi M, Mirzaii-Sousefidi R. The comparison of apotel plus low dose of morphine and full dose of morphine in pain relief in patients with acute renal colic. Addict Health 2015; 7(1-2): 66-73.

18. Masoumi K, Forouzan A, Asgari Darian A, Feli M, Barzegari $\mathrm{H}$, Khavanin A. Comparison of clinical efficacy of intravenous acetaminophen with intravenous morphine in acute renal colic: a randomized, doubleblind, controlled trial. Emerg Med Int 2014; 2014: 571326. doi: 10.1155/2014/571326.

19. Grissa MH, Claessens YE, Bouida W, Boubaker H, Boudhib L, Kerkeni W, et al. Paracetamol vs piroxicam to relieve pain in renal colic. Results of a randomized controlled trial. Am J Emerg Med 2011; 29(2): 203-6. doi: 10.1016/j.ajem.2009.09.019.
20. Hyllested M, Jones S, Pedersen JL, Kehlet H. Comparative effect of paracetamol, NSAIDs or their combination in postoperative pain management: a qualitative review. $\mathrm{Br}$ J Anaesth 2002; 88(2): 199-214.

21. Azizkhani R, Pourafzali SM, Baloochestani E, Masoumi B. Comparing the analgesic effect of intravenous acetaminophen and morphine on patients with renal colic pain referring to the emergency department: A randomized controlled trial. J Res Med Sci 2013; 18(9): 772-6.

22. Serinken M, Eken C, Turkcuer I, Elicabuk H, Uyanik E, Schultz $\mathrm{CH}$. Intravenous paracetamol versus morphine for renal colic in the emergency department: a randomised double-blind controlled trial. Emerg Med J 2012; 29(11): 902-5. doi: 10.1136/emermed-2011-200165. 